\title{
Anatomía foliar comparativa de tres especies de Furcraea (Asparagaceae: Agavoideae)
}

\author{
(D) Jhon Steven Murillo-Serna ${ }^{1,2}$, Edgar Javier Rincón-Barón ${ }^{1}$ y Fernando Alzate-Guarin ${ }^{1}$
}

Recibido: 25.01.2018; aceptado: 8.08.2018

\begin{abstract}
Comparative leaf anatomy of three species of Furcraea (Asparagaceae: Agavoideae)). The Neotropical genus Furcraea Vent. is widely cultivated to produce fibers that are known as Fique or Cabuya. A histological analysis of leaves from three species of Furcraea cultivated in Colombia was performed in order to make comparative descriptions among them. Different sections of mature leaves ready to be harvested were processed using conventional histological techniques. The three species showed homogeneous anatomical features, some of which were: one-layered epidermis, thick cuticle, encrypted stomata, undifferentiated mesophyll, the presence of calcium oxalate crystals, septate sclerenchyma fibers, associated or stranded fiber bundles, and brachysclereids associated with the leaf margin. Apart from the brachysclereids, there is no histological character that allowed a clear differentiation among the three studied cultivars. The anatomy of these plants is also discussed according to their photosynthetic metabolism.
\end{abstract}

Keywords: cabuya, fique, plant tissues, xerophytic plants.

RESUMEN - (Anatomía foliar comparativa de tres especies de Furcraea (Asparagaceae: Agavoideae)). El género neotropical Furcraea Vent. es ampliamente cultivado para la producción de fibras conocidas como Fique o Cabuya. Se realizó un análisis histológico de las hojas de tres especies cultivadas en Colombia, con la finalidad de realizar la descripción comparativa entre ellas. Diferentes secciones de hojas listas para su cosecha fueron procesadas utilizando técnicas histológicas convencionales. Las tres especies presentaron características anatómicas comparativamente muy homogéneas dentro de las que resaltan: epidermis monoestratificada, cutícula gruesa, estomas encriptados, mesófilo no diferenciado, presencia de cristales de oxalato de calcio, fibras esclerenquimáticas de tipo septado asociadas a los haces vasculares o formando cordones y braquiesclereidas asociadas al margen foliar. Estas últimas varían ligeramente entre las especies estudiadas en el lumen, la forma de las punteaduras y el grado de lignificación, lo cual parece estar correlacionado con la dureza del margen foliar. Aparte de las braquiesclereidas, no se advierten suficientes caracteres histológicos que permitan la diferenciación clara entre los cultivares estudiados. Se discute también la anatomía de estas plantas en función de su metabolismo fotosintético.

Palabras clave: cabuya, fique, tejidos vegetales, xerofitas

\section{Introducción}

El género Furcraea Vent. (Asparagaceae) está compuesto por cerca de 25 especies que ocurren de forma natural en el neotrópico y naturalizadas en Sudáfrica, Estados Unidos, Portugal, Tailandia, India, Australia e islas Oceánicas (García-Mendoza 2000, Barbosa et al. 2017). Aunque el género se encuentra bien delimitado taxonómicamente respecto a otros géneros cercanos filogenéticamente, este presenta problemas de circunscripción entre sus especies (García-Mendoza 2000). Furcraea fue incluido en la familia Agavaceae del orden Liliales por Cronquist
(1981), mientras que Takhtajan (2009) lo incluyó en la misma familia, pero circunscrito en el orden Amaryllidales. APG IV (2016) incluye al género en la familia Asparagaceae, subfamilia Agavoideae, del orden Asparagales, basados fundamentalmente en caracteres moleculares.

Las plantas del género Furcraea son popularmente conocidas por su uso económico, debido a que son productoras de fibras usadas en la manufactura de diversos elementos como artesanías, vestuarios o empaques (Peinado et al. 2006, Guevara \& Vallejo 2014). Adicionalmente en diferentes especies del

1. Instituto de Biología, Herbario HUA, Universidad de Antioquia. Calle 67 No 53-108. Medellín, Colombia

2. Autor para correspondência: murillojsteven@gmail.com 
género se han encontrado importantes potencialidades económicas como: moléculas con actividad farmacológica (Yokosuka et al. 2009, Benavides et al. 2012), insecticidas (Castellanos et al. 2011, Iannacone 2013, Fernández et al. 2015, Sobrino et al. 2016), fungicidas (Solarte et al. 2012, Solarte \& Osorio 2014), herbicidas (Osorio-Salazar et al. 2009), potenciadores de la durabilidad y resistencia de materiales como el hormigón (Ochoa \& Jaramillo 2007) y potencial en biorremediación (Lozano-Rivas 2011, 2012, Rivera et al. 2012, Baldeon 2013, Ríos et al. 2017). Debido al potencial que presentan estas plantas se han establecido además protocolos de propagación (Martínez \& Pacheco 2006) y se han realizado estudios metodológicos para potencializar el efecto plaguicida de sus extractos (La Torre et al. 2013), así como pruebas sobre la resistencia de las fibras con aplicaciones en la industria (Gañan \& Mondragón 2002).

Algunas especies del género Furcraea también han sido objeto de estudios taxonómicos (Baker 1888, Drummond 1907, Álvarez 1996, García-Mendoza 2000), filogenéticos (Bogler \& Simpson 1995, 1996) y fisiológicos (Casierra-Posada \& Gómez 2008, Casierra-Posada \& González 2009). En cuanto al estudio de su anatomía, se destacan los trabajos de Blunden \& Jewers (1973) y Álvarez (1990) en donde describen los complejos estomáticos en diferentes géneros de la subfamilia Agavoideae mostrando que la anatomía que exhiben géneros como Agave L. y Beschorneria Kunth es muy similar a Furcraea, pero que difiere marcadamente respecto a otros géneros de la subfamilia.

En este trabajo se describe de forma comparativa la anatomía de tres especies cultivadas presentes en Colombia, Furcraea cabuya Trel., Furcraea gigantea Vent. y Furcraea macrophylla Baker, con la finalidad de explorar diferencias en la composición anatómica de estas especies, que puedan aportar caracteres para la determinación taxonómica de estas y que sirvan como base para posteriores estudios fisiológicos o comparativos en el área de la sistemática de plantas.

\section{Materiales y métodos}

Material vegetal - Seis hojas maduras de seis individuos diferentes para cada uno de los tres cultivares de Furcraea se recolectaron en fincas productoras, ubicadas en los departamentos de Antioquia y Nariño (Colombia). Los cultivares fueron relacionados por los productores como: Ceniza, Negra Común y Uña de Águila, los cuales fueron identificados con base en literatura taxonómica y colecciones de herbario como Furcraea cabuya Trel., Furcraea gigantea Vent. y Furcraea macrophylla Baker respectivamente. Las colecciones botánicas testigo fueron depositadas en el Herbario de la Universidad de Antioquia (HUA) bajo los códigos de herbario 213358, 213359, 213360 , 213361 y 213362.

Las hojas fueron seccionadas, fragmentadas y preservadas en fijador FAA (formol-alcohol-ácido acético) durante tres días y posteriormente fueron almacenadas en solución de mantenimiento y ablandamiento (Etanol-Glicerol).

Preparación de placas - La lamina foliar fue estudiada en tres segmentos (base, medio y ápice) y cada uno de estos, a su vez, se dividió en dos regiones (medial y distal), de donde se obtuvo fracciones de las caras abaxiales, adaxiales y centrales de la hoja. Las fracciones de las hojas previamente fijadas y preservadas, fueron seccionadas en fragmentos de $0.5 \mathrm{~cm}$ de longitud aproximadamente, las cuales fueron deshidratadas en una serie de alcoholes en grado ascendente siguiendo el protocolo de Ruzin (1999) con modificaciones realizadas por los autores. Las modificaciones consistieron en un aclaramiento inicial con Histochoice ${ }^{\circledR}$, y posterior embebido en una mezcla de Histochoice $\AA /$ parafina en una serie donde se aumentaron las proporciones de parafina hasta embeber finalmente en parafina pura durante 16 horas a $55^{\circ} \mathrm{C}$.

Se tomaron secciones longitudinales y transversales, con grosores de 8-10 $\mu \mathrm{m}$ en micrótomo rotatorio Leica RM2125 armado con cuchillas de perfil estrecho Leica 819, para ser posteriormente coloreadas. Las placas con los cortes anatómicos fueron observadas en un microscopio óptico Olympus $\mathrm{BH}-2$ y las fotografías fueron tomadas utilizando cámara digital Olympus E-620.

Coloración e histoquímica - Las secciones obtenidas fueron coloreadas con Safranina O y Alcian Blue según el protocolo de Tolivia y Tolivia (1987). Para descripciones adicionales las secciones también fueron coloreadas con la tinción del ácido periódico de Schiff (PAS) y Toluidina acida según Yeung \& Saxena (2005) y Ruzin (1999).

Las descripciones anatómicas se realizaron según la terminología propuesta por Alonso (2011), Dickison (2000), Evert (2006), Roth (1976) y el Leaf Architecture Working Group (1999). 


\section{Resultados}

En las tres especies de Furcraea Vent. estudiadas, la composición anatómica en los segmentos de la hoja (base, medio y ápice) y en las regiones mediales y distales, son muy homogéneas entre sí en cuanto a la estructura y composición de los tejidos. La única diferencia observable entre estas porciones se encuentra en el grosor de la hoja y el número de paquetes de fibras esclerenquimáticas, los cuales ocurren con mayor abundancia hacía las porciones basales de la lámina foliar, es decir, en las porciones de mayor calibre.

Epidermis - La epidermis es monoestratificada tanto en la cara adaxial como en la abaxial y está compuesta por células columnares y campanuladas que ocasionalmente se prolongan formando protuberancias piramidales. Las células epidérmicas se caracterizan además por presentar un engrosamiento variable en sus paredes, siendo más gruesa la pared periclinal externa que se fusiona con una cutícula engrosada (figuras $1 \mathrm{a}, \mathrm{b}$ ). La cutícula es uniforme en la cara adaxial de las hojas (figura 1d); en tanto que en la cara abaxial presenta plegamientos y crestas papiladas (figuras 1e, f), las cuales no se encontraron en los márgenes foliares (figuras $3 \mathrm{~g}, \mathrm{~h}, \mathrm{i}$ ). La epidermis foliar de los especímenes estudiados carecía de tricomas.

Las hojas son anfiestomáticas (figura 2a) y el patrón estomático es paratetracítico, con dos células subsidiarias alargadas laterales y paralelas a las células de guarda y dos células subsidiarias polares estrechas (figura 1c). En las tres especies los estomas son encriptados y estos se comunican internamente con el parénquima fotosintético por medio de una cámara subestomática amplia. Se observa además que, en la superficie de la cripta, las células anexas sobrepasan a las células oclusivas y están cubiertas por cutícula formando dos proyecciones a manera de cuernos, generalmente más pronunciados en la cara abaxial (figuras 1g, h, i). De igual forma, el borde externo e interno de las células oclusivas se encuentra cubierto por cutina formando cuernos de menor tamaño (figura $1 \mathrm{~g})$.

Mesófilo - No existe diferenciación en los tejidos del mesófilo, por lo que no se reconoce parénquima de empalizada ni esponjoso. El mesófilo es isolateral con parénquima homogéneo interrumpido solamente por la vascularización de la hoja (figura 2a). Las células parenquimatosas son más o menos isodiamétricas con cloroplastos abundantes hacia las zonas epidérmicas en ambas caras de la hoja (figuras 4e, f) y muy escasos o ausentes hacía el centro de la hoja. En la parte central las células parenquimáticas son más grandes y de contorno redondeado (figura $2 b$ ). El mesófilo de la hoja presenta inclusiones citoplasmáticas en forma de cristales prismáticos (figura 2c, f), estiletes (figura 2d) y paquetes de rafidios (figura 2e).

Tejidos vasculares - Los haces vasculares se distribuyen de forma homogénea y hacia la región medial del mesófilo (figura 2a), variando en tamaño (figuras 3a, b) y presentando una estructura básica similar, la cual está conformada por un paquete de células de xilema ligeramente lignificadas asociado a otro paquete de floema, generalmente de menor tamaño (figuras $3 \mathrm{~d}$, e). Estos haces pueden estar parcialmente rodeados por casquetes de fibras esclerenquimáticas (figuras $3 a, d)$ o inmersos en vainas de esclerénquima en los haces vasculares de menor tamaño (figuras $3 \mathrm{~b}$, e). El tamaño de los haces vasculares es mayor hacia la región central del mesófilo y disminuyen en tamaño hacia las regiones periféricas. En cortes longitudinales se observa que las fibras esclerenquimáticas son del tipo septado y en el mesófilo forman paquetes compactos de diferentes tamaños (figuras 2a,g-i, 3b). Estas fibras septadas presentan tabiques transversales de pared primaria, además de abundantes puntuaciones (figuras 2g-i). En las secciones transversales las fibras exhiben un lumen amplio y generalmente circular con engrosamientos de pared secundaria muy marcados (figuras 3c, f).

Hacia el margen de la hoja, el mesófilo deriva en braquiesclereidas, estas estructuras solo se observaron en esta zona de la lámina foliar y presentan formas ligeramente variables en cada una de las especies. Según lo observado en este estudio, la variación se encuentra en el grado de lignificación del margen foliar y este parece estar asociado a la producción de espinas. Furcraea cabuya es la especie menos lignificada en el margen, lo que concuerda con sus hojas desprovistas de espinas, donde se observaron braquiesclereidas con paredes engrosadas por pared secundaria y primaria, lúmenes irregulares y puntuaciones simples (figuras $3 \mathrm{~g}$, j). Furcraea gigantea presenta mayor concentración de esclereidas en el margen foliar y estas conforman un esclerénquima muy similar al que se produce en pequeñas porciones en las zonas más lignificadas de $F$. cabuya (figura 3j-k). Sin embargo, en esta especie el lumen es amplio, claramente delimitado y de contorno circular, con paredes segundarias marcadamente engrosadas y con punteaduras simples en forma de 


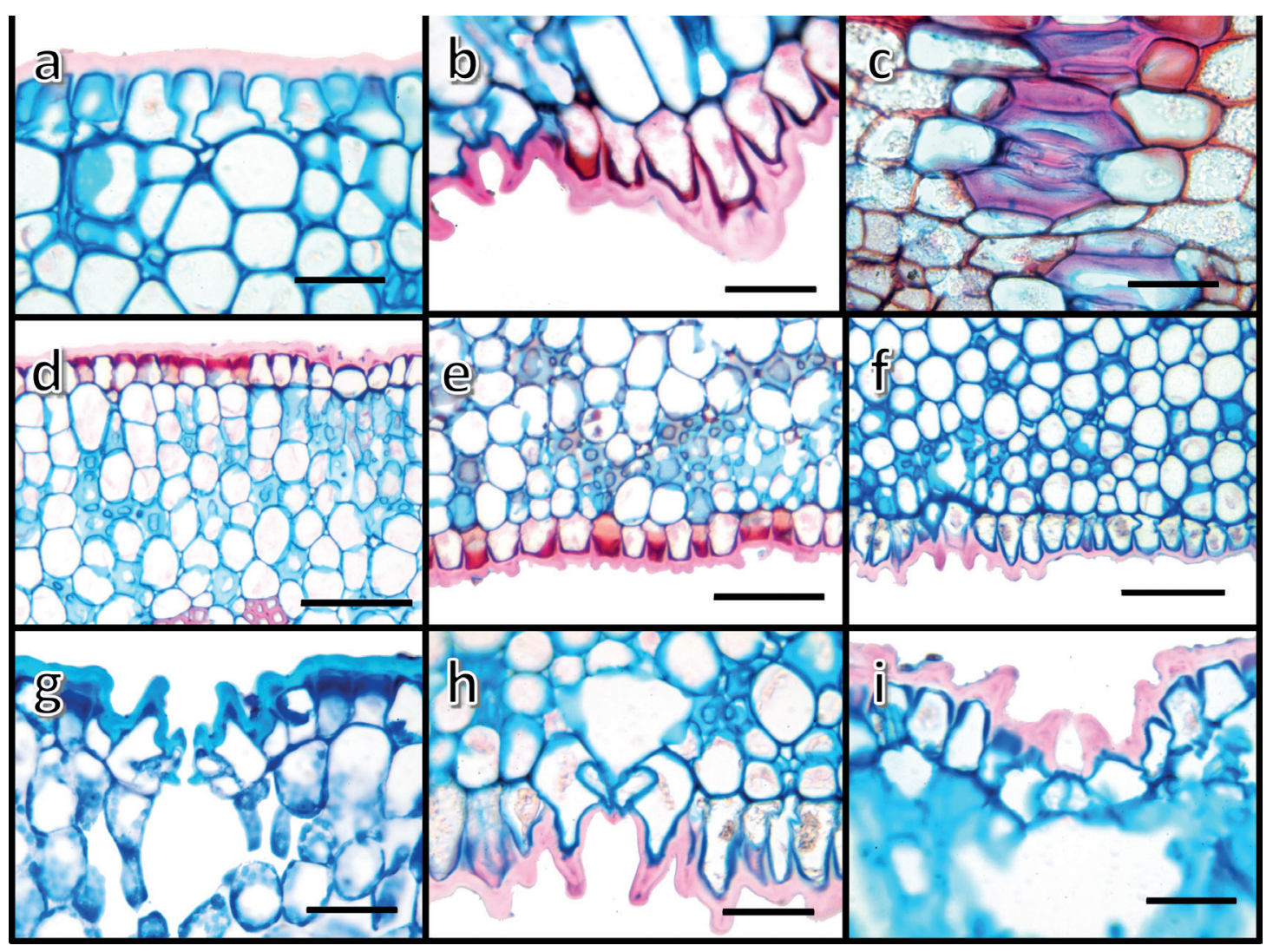

Figura 1. Secciones transversales en el segmento medio de las hojas de Furcraea cabuya (FC), F. gigantea (FG) y F. macrophylla (FM) (a-b, d-i) y sección paradérmica de FC (c). a. Epidermis y cutícula en la cara adaxial de FC. b. Células epidérmicas columnares alargadas y contiguas al aparato estomático en FC. c. Estoma en vista superficial de la cara adaxial en FC. d. Epidermis y cutícula en la cara adaxial de FC. e. Epidermis y cutícula en la cara abaxial de FG. f. Epidermis y cutícula en la cara abaxial de FM. g. Aparato estomático en la cara adaxial de FC. h. Aparato estomático en la cara abaxial de FG. i. Aparato estomático en la cara adaxial de FM. Tinciones: Safranina O-Alcian Blue: a-f y h-i; Toluidina O: g. Escala: a-c $=50 \mu \mathrm{m}$, d-f $=100 \mu \mathrm{m}, \mathrm{g}-\mathrm{i}=50 \mu \mathrm{m}$.

Figure 1. Transverse sections in the middle segment of the leaves of Furcraea cabuya (FC), F. gigantea (FG) and F. macrophylla (FM) (a-b, d-i) and paradermic section of FC (c). a. Epidermis and cuticle on the adaxial surface of FC. b. Elongated columnar epidermic cells adjacent to the stomatal apparatus in FC. c. Adaxial stoma of FC in surface view. d. Epidermis and cuticle on the adaxial surface of FC. e. Epidermis and cuticle on the abaxial surface of FG. f. Epidermis and cuticle on the abaxial surface of FM. g. Stomatal apparatus on the adaxial surface of FC. h. Stomatal apparatus on the abaxial surface of FG. i. Stomatal apparatus on the adaxial surface of FM. Stains used: Safranin O-Alcian Blue: a-f and h-i; Toluidine Blue O: g. Scale: a-c $=50 \mu \mathrm{m}, \mathrm{d}-\mathrm{f}=100 \mu \mathrm{m}, \mathrm{g}-\mathrm{i}=50 \mu \mathrm{m}$.

canalículos (figuras 3h, k). Furcraea macrophylla posee esclereidas alargadas hacia las partes más distales del margen de la hoja, las cuales son comparativamente de mayor tamaño y presentan fuertes engrosamientos de pared secundaria con lúmenes consistentemente más pequeños y puntuaciones en canalículos en su mayoría simples y ocasionalmente ramificados (figuras $3 i, 1)$. En las especies F. macrophylla y F. gigantea, las braquiesclereidas son las principales constituyentes de las espinas (figura 3i).

Histoquímica - Mediante la tinción de PAS se evidenciaron diferencias en la composición de química celular, lo cual se advierte en las intensidades de coloración. Se obtuvo un mayor contraste en la coloración de estructuras compuestas por celulosa como paredes celulares, mientras que no se detectaron gránulos de almidón en ninguna región de la hoja mediante la coloración de PAS. Esta tinción mostró resultados positivos en los cloroplastos, indicando la presencia de carbohidratos insolubles entre los que se encuentra el almidón. Se observó también que las zonas epidérmicas y el floema presentan mayor intensidad con la tinción de PAS (figuras 4a-d).

La tinción metacromática con Toluidina $\mathrm{O}$, muestra el nivel de diferenciación celular entre los diferentes tejidos que componen la hoja, que, para el caso de los especímenes estudiados, presentan alto nivel de lignificación hacia la epidermis de la hoja y abundantes contenidos celulares (figuras 1g, 2d, e). Hacia el centro de la lámina foliar se observa un menor engrosamiento de las paredes 


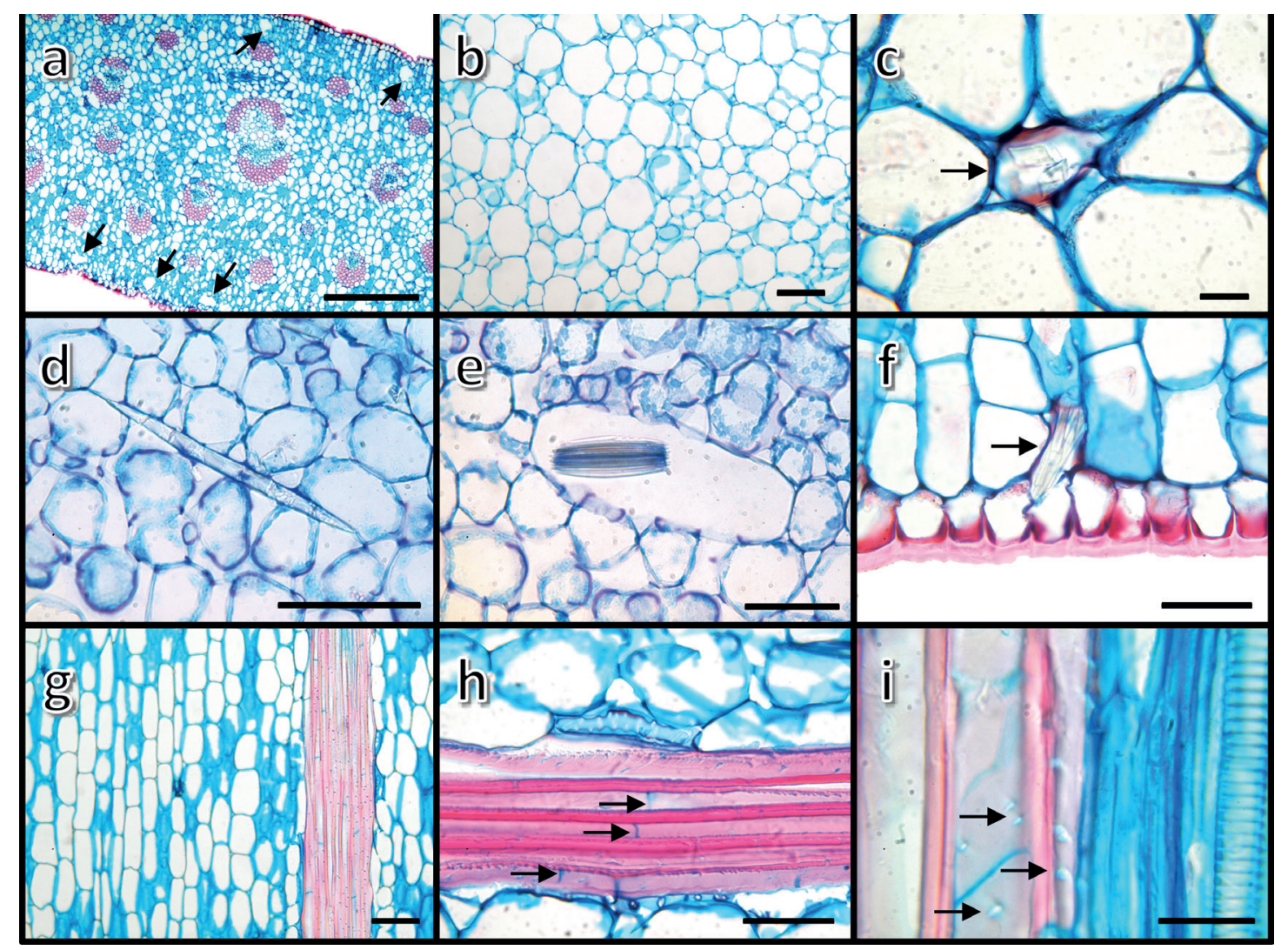

Figura 2. Secciones transversales (a-f) y longitudinales del segmento medio y región medial-central (g-i) de las hojas de Furcraea cabuya (FC) y F. gigantea (FG). a. Ubicación de los aparatos estomáticos (flechas) y distribución de la vascularización en el segmento apical y la región medial de la hoja en FC. b. Parénquima en el segmento de la base y la región medial-central de la lámina foliar en FC. c. Inclusiones citoplasmáticas en forma de cristales prismáticos (flecha) en FC. d. Inclusión citoplasmática en forma estiloide en FG. e. Inclusiones citoplasmáticas en paquete de rafidios en FG. f. Inclusiones citoplasmáticas en forma de cristales prismáticos contiguos a la epidermis (flecha) en FC. g. Cordón de fibras esclerenquimáticas septadas y parénquima asociado en FC. h. Detalle de los septos de pared primaria en las fibras esclerenquimáticas (flechas) y parénquima asociado en FC. i. Detalle del septo de pared primaria y las punteaduras (flechas) en una fibra esclerenquimática septada y xilema anular en FC. Tinciones: Safranina O-Alcian Blue: a-c y f-i; Toluidina O: d-e. Escala: $\mathrm{a}=500 \mu \mathrm{m}, \mathrm{b}=100 \mu \mathrm{m}, \mathrm{c}=10 \mu \mathrm{m}, \mathrm{d}=300 \mu \mathrm{m}, \mathrm{e}=200 \mu \mathrm{m}, \mathrm{f}=50 \mu \mathrm{m}, \mathrm{g}=100 \mu \mathrm{m}, \mathrm{h}=50 \mu \mathrm{m}, \mathrm{i}=20 \mu \mathrm{m}$.

Figure 2. Transverse (a-f) and longitudinal (g-i) sections of the middle segment and median-central region of the leaves of Furcraea cabuya (FC) and F. gigantea (FG). a. Location of the stomatal apparatus (arrows) and distribution of leaf vascularization in the apical segment and the median region of the leaf in FC. b. Parenchyma at the basal segment and the median-central region of the leaf in FC. c. Prismatic-shaped cytoplasmic inclusions (arrow) in FC. d. Styloid-shaped cytoplasmic inclusions in FG. e. Raphide-shaped bundles in FG. f. Prismatic crystal-shaped cytoplasmic inclusions contiguous to the epidermis (arrow) in FC. g. Septate fiber bundle and associated parenchyma in FC. h. Detail of the primary wall septa in the sclerenchyma fibers (arrows) and associated parenchyma in FC. i. Detail of a primary wall septum and pits (arrows) in a septate sclerenchyma fiber and annular xylem in FC. Stains used: Safranin O-Alcian Blue: $\mathrm{a}-\mathrm{c}$ and f-i; Toluidine Blue O: d-e. Scale: $\mathrm{a}=500 \mu \mathrm{m}, \mathrm{b}=100 \mu \mathrm{m}, \mathrm{c}=10 \mu \mathrm{m}, \mathrm{d}=300 \mu \mathrm{m}, \mathrm{e}=200 \mu \mathrm{m}, \mathrm{f}=50 \mu \mathrm{m}, \mathrm{g}=100 \mu \mathrm{m}$, $\mathrm{h}=50 \mu \mathrm{m}, \mathrm{i}=20 \mu \mathrm{m}$.

celulares y una marcada disminución o ausencia de los contenidos celulares. Sin embargo, en los tejidos menos diferenciados es clara la unión entre la lámina media con las paredes secundarias aun no diferenciadas, lo cual también se observa en las braquiesclereidas del margen foliar, mostrando así, que incluso las células de parénquima presentan altos grados de lignificación.

La tinción de PAS combinada con Amidoblack se usó para determinar la presencia de proteínas en los tejidos de la hoja, y con esto se encontró una mayor acumulación de proteínas en las porciones del mesófilo que se encuentran próximas a la epidermis y al floema. En la región central de la hoja, que está compuesta por parénquima acumulador, no se observó una reacción notable con esta tinción (figura 4e, f).

\section{Discusión}

La anatomía foliar de las especies de Furcraea incluidas en este estudio es consistente con la 


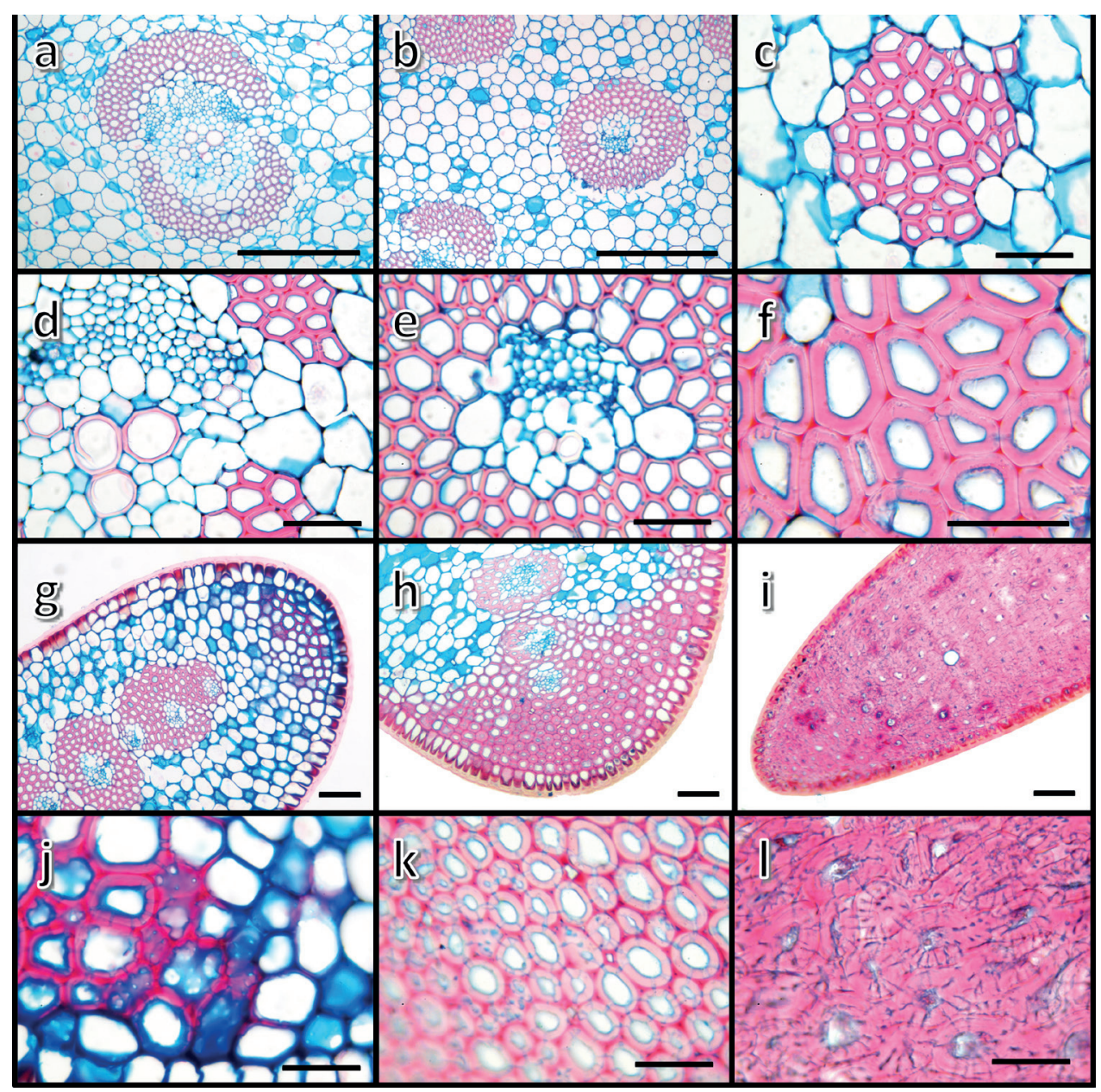

Figura 3. Secciones transversales de las hojas de Furcraea cabuya (FC), F. gigantea (FG) y F. macrophylla (FM). Segmento medio y región medial (a-f); segmento apical y región distal (g-l). a. Haz vascular con casquetes de fibras esclerenquimáticas en FC. b. Haz vascular con vaina de esclerénquima y paquete de fibras esclerenquimáticas en FC. c. Cordón de fibras septadas en FC. d. Xilema, floema, esclerénquima y parénquima asociado a un haz vascular en FC. e. Xilema y floema rodeado por vaina de esclerénquima en FC. f. Detalle de las fibras esclerenquimáticas empaquetadas en FC. g. Margen foliar en FC. h. Margen foliar en FG. i. Espina del margen foliar en FM. j. Braquiesclereidas del margen foliar en FC. k. Braquiesclereidas del margen foliar en FG. 1. Braquiesclereidas del margen foliar en FM. Tinciones: Safranina O-Alcian Blue: a-l. Escala: $\mathrm{a}-\mathrm{b}=300 \mu \mathrm{m}, \mathrm{c}-\mathrm{e}=50 \mu \mathrm{m}, \mathrm{f}=30 \mu \mathrm{m}, \mathrm{g}-\mathrm{i}=100 \mu \mathrm{m}, \mathrm{j}-1=50 \mu \mathrm{m}$.

Figure 3. Transverse sections of the leaves of Furcraea cabuya (FC), F. gigantea (FG) and F. macrophylla (FM). Middle segment and median region (a-f); Apical segment and distal region (g-1). a. Vascular bundle with sclerenchyma fiber caps in FC. b. Vascular strand with sclerenchyma sheath and sclerenchyma fiber bundle in FC. c. Septate fiber bundle in FC. d. Xylem, phloem, sclerenchyma and parenchyma associated with a vascular bundle in FC. e. Xylem and phloem surrounded by sclerenchyma sheath in FC. f. Detail of bundled sclerenchyma fibers in FC. g. Leaf margin in FC. h. Leaf margin in FG. i. Leaf margin spine in FM. j. Leaf margin brachysclereids in FC. $k$. Leaf margin brachysclereids in FG. 1. Leaf margin brachysclereids in FM. Stains used: Safranin O-Alcian Blue: a-1. Scale: a-b= $300 \mu \mathrm{m}, \mathrm{c}-\mathrm{e}=50 \mu \mathrm{m}, \mathrm{f}=30 \mu \mathrm{m}, \mathrm{g}-\mathrm{i}=100 \mu \mathrm{m}, \mathrm{j}-1=50 \mu \mathrm{m}$.

conformación histológica de tipo xeromórfico que se observa en las plantas adaptadas a la sequía, déficit de nutrientes en el suelo y a niveles altos de radiación (Andersen et al. 2006). Dentro de estos caracteres xeromórficos se encuentran: paredes engrosadas en las células epidérmicas, cutícula gruesa y aparatos estomáticos encriptados en la epidermis, lo cual se encontró en las especies de Furcraea. Estas particularidades histológicas han sido interpretadas como una estrategia de las plantas xerofíticas para hacer frente a la deshidratación excesiva, aunque, este síndrome también se puede encontrar en especies adaptadas a suelos pobres en nutrientes y de áreas inundables (Dickison 2000). Otra adaptación notable en el contexto ecológico es la abundancia de parénquima acumulador de agua en la región central de la hoja, con células de paredes delgadas pero reforzadas y desprovisto de otras sustancias, según las 


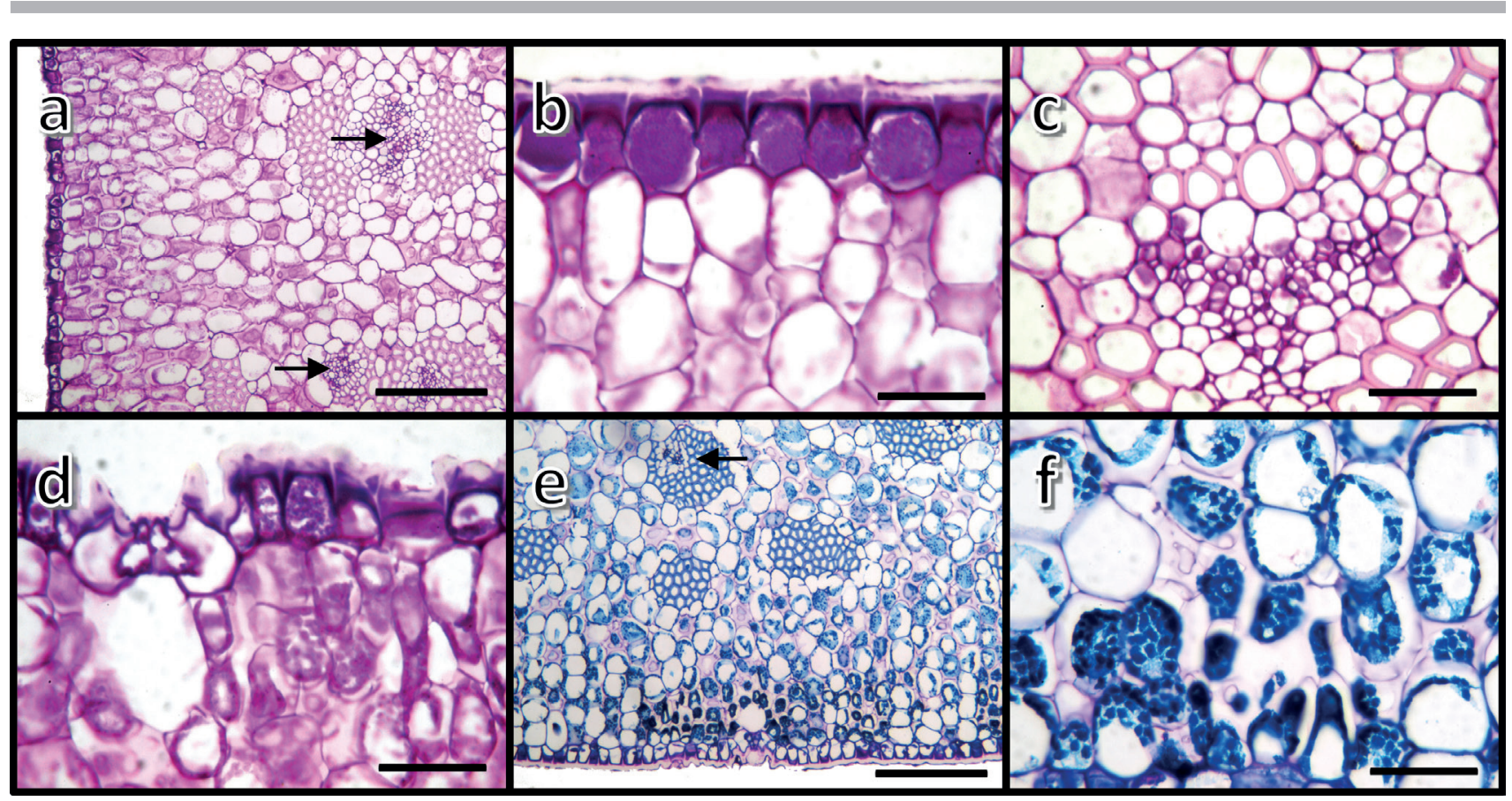

Figura 4. Secciones transversales de las hojas de Furcraea cabuya en el segmento apical y región medial. a. Mesófilo detallando el floema (flechas) y vascularización. b. Detalle de la epidermis adaxial. c. Detalle del haz vascular. d. Aparato estomático. e. Mesófilo detallando el floema (flechas) y vascularización. f. Parénquima fotosintético. Tinciones: PAS: a-d; PAS y Amidoblack: e-f. Escala: a = 200 $\mu \mathrm{m}, \mathrm{b}-\mathrm{d}=$ $50 \mu \mathrm{m}, \mathrm{e}=200 \mu \mathrm{m}, \mathrm{f}=50 \mu \mathrm{m}$.

Figure 4. Transverse sections of the leaves of Furcraea cabuya (FC) in the apical segment and median region. a. Mesophyll, highlighting the phloem (arrows) and vascularization. b. Detail of the adaxial epidermis. c. Detail of a vascular bundle. d. Stomatal apparatus. e. Mesophyll, highlighting the phloem (arrow) and vascularization. f. Photosynthetic parenchyma. Stains used; PAS: a-d; PAS and Amidoblack; e-f. Scale: $\mathrm{a}=200 \mu \mathrm{m}, \mathrm{b}-\mathrm{d}=50 \mu \mathrm{m}, \mathrm{e}=200 \mu \mathrm{m}, \mathrm{f}=50 \mu \mathrm{m}$.

pruebas histoquímicas realizadas para carbohidratos y proteínas. Es importante mencionar que el proceso de fijación utilizado en estas pruebas puede limpiar o degradar estas sustancias químicas, ocasionando errores en los resultados obtenidos. Todo esto junto a la ocurrencia de esclerofilia, ayuda a soportar los cambios en las presiones de turgencia y mantener la estructura coriácea de la hoja, evitando así el colapso de esta (Dickison 2000). La ocurrencia de hojas con mesófilo indiferenciado y compacto, está demostrando la adaptación de estos taxones para soportar una alta radiación solar, lo cual ha sido planteado también para Lippia turbinata Griseb. por Andersen et al. (2006).

Desde el punto fisiológico muchas de las plantas suculentas que crecen en hábitats cálidos y áridos presentan una variante bioquímica temporal en la fijación del $\mathrm{CO}_{2}$ conocida como Metabolismo Acido de las Crassulaceae (CAM). Las especies de Furcraea estudiadas presentan la estructura CAM descrita por Alché et al. (2010), la cual consiste en el desarrollo de hojas gruesas con un mesófilo indiferenciado, de células notablemente vacuoladas y con cloroplastos periféricos. Heyduk et al. (2016) describen la importancia de la anatomía en la aparición evolutiva del metabolismo CAM en la subfamilia Agavoideae, mostrando que esta innovación metabólica apareció en momentos diferentes, durante la historia evolutiva de este linaje, que se encuentra representado además por taxones con metabolismo C3. Aunque Heyduk et al. (2016) realizaron una amplia revisión taxonómica de esta subfamilia, no incluyeron ninguna especie del género Furcraea en su análisis y la naturaleza bioquímica de la fotosíntesis de este género sigue siendo desconocida. Sin embargo, la anatomía descrita en este trabajo, sumado a los estudios previos de Casierra \& Gonzales (2009) y Barbosa et al. (2017) sugieren que estas plantas presentan metabolismo fotosintético CAM.

Respecto a estudios anatómicos precedentes a este trabajo, se destacan las descripciones de los patrones estomáticos en especies del género Furcraea, incluyendo a F. macrophylla, realizadas por Álvarez (1990), las cuales fueron coherentes con lo encontrado en este estudio. En el trabajo clásico de Blunden \& Jewers (1973) se describe la anatomía foliar de 40 especies de la subfamilia Agavoideae incluyendo 
tres del género Furcraea (F. cabuya, F. gigantea y $F$. selloa K. Koch), lo observado en esta investigación es concordante con las descripciones precedentes. Las braquiesclereidas que ocurren en Furcraea no habían sido descritas anteriormente y parecen estar asociadas a la lignificación de las hojas en el margen foliar. Son evidentes las diferencias en cuanto a la morfología y grado de lignificación de las braquiesclereidas, lo cual constituye un carácter taxonómico útil para la identificación de las especies aquí estudiadas. Esta característica no fue variable a nivel de especie, entre los especímenes descritos, sin embargo, es necesario realizar estudios posteriores con las demás especies del género para determinar con certeza el grado de utilidad de este carácter en la determinación y delimitaciones de especies.

En este trabajo se describió la anatomía foliar de tres especies del género Furcraea, donde se observa que la construcción y composición anatómica de las láminas foliares es muy similar entre sí, encontrándose variaciones relevantes en la morfología de las braquiesclereidas, razón por la cual se sugiere la realización de estudios comparativos enfocados en estas estructuras para evaluar su relación con el desarrollo de espinas foliares y su variación en un contexto ecológico y taxonómico. La anatomía fisiológica de estas plantas corresponde a la descrita previamente para especies con metabolismo fotosintético CAM, sin embargo, hay que realizar investigaciones cuyo enfoque permita resolver esta pregunta.

A pesar de que estas especies constituyen una fuente comercial tradicional de fibras naturales y de representar un rubro económico importante, solo se tienen descripciones anatómicas parciales para 8 especies, de las cerca de 25 especies reconocidas en Furcraea y de la considerable cantidad de variedades y cultivares.

\section{Agradecimientos}

Los autores expresan sus agradecimientos a la Corporación Colombiana de Investigación Agropecuaria (CORPOICA) por la financiación obtenida para llevar a cabo esta investigación. Al personal del Herbario de la Universidad de Antioquia (HUA) por facilitarnos el acceso a sus colecciones botánicas y referencias bibliográficas. A Isabel Carmona Gallego (GEOBOTA) por sus comentarios y aportes.

\section{Literatura citada}

Alché, J de D., Olmedilla, A. \& Rodríguez, M.I. 2010. Análisis estructural del aparato fotosintético en plantas C4 y CAM mediante microscopía de fluorescencia/ confocal. En: J.L. González, A. Chueca (eds.). C4 y CAM. Características generales y uso en programas de desarrollo de tierras áridas y semiáridas. Consejo Superior de Investigaciones Científicas. Fundación Ramón Areces, Taravilla, pp. 115-128.

Alonso, J.R. 2011. Manual de histología vegetal. Ediciones Mundi-Prensa, Madrid.

Álvarez, A. 1990. El complejo estomático en la familia Agavaceae II. Epidermis adulta. Feddes Repertorium 101: 113-134.

Álvarez, A. 1996. El género Furcraea (Agavaceae) en Cuba. Anales del Instituto de Biología Serie Botánica 67: 329-346.

Andersen, A., Lucchini, F., Moriconi, J. \& Fernández, E.A. 2006. Variabilidad en la morfo-anatomía foliar de Lippia turbinata (Verbenaceae) en la provincia de San Luis (Argentina). Phyton 75: 137-143.

APG IV. 2016. An update of the Angiosperm Phylogeny Group classification for the orders and families of flowering plants: APG IV. Botanical Journal of the Linnean Society 181: 1-20.

Baker, J.G. 1888. Handbook of the Amaryllideae, including the Alstroemerieae and Agaveae. Bell, London.

Baldeon, J.E. 2013. Estudio de retención de metales pesados en aguas sintéticas (preparadas en el laboratorio) utilizando como lecho filtrante la fibra de cabuya Furcraea andina como alternativa de biorremediación. Tesis de Pregrado, Escuela de Ingeniería Ambiental, Universidad Nacional de Chimborazo, Chimborazo.

Barbosa, C., Otalora, J.M., Giehl, E.L.H., Villalobos, F., Loyola, R., Tessarolo, G., Machado, N. \& Castellani, T.T. 2017. Changes in the realized niche of the invasive succulent CAM plant Furcraea foetida. Austral Ecology 42: 643-654.

Benavides, O.L, Arango, O., Hurtado, A.M. \& Rojas, M.C. 2012. Cuantificación de Sapogeninas del Jugo Fresco y Fermentado de Fique (Furcraea gigantea) mediante cromatografía liquida de alta resolución (HPLC-PDA). Información Tecnológica 23: 67-76.

Blunden, Y. \& Jewers, K. 1973. The comparative leaf anatomy of Agave, Beschorneria, Doryanthes and Furcraea species (Agavaceae: Agaveae). Botanical Journal of the Linnean Society 66: 157-179.

Bogler, D.J. \& Simpson, B.B. 1995. A chloroplast DNA study of the Agavaceae. Systematic Botany 20: 191-205.

Bogler, D.J. \& Simpson, B.B. 1996. Phylogeny of Agavaceae based on ITS rDNA sequence variation. American Journal of Botany 83: 1225-1235.

Casierra-Posada, F. \& Gómez, N.E. 2008. Crecimiento foliar y radical en plantas de fique (Furcraea castilla y $F$. macrophylla) bajo estrés por encharcamiento. Agronomía Colombiana 26: 381-388. 
Casierra-Posada, F. \& González, D. 2009. Cambio circadiano de $\mathrm{pH}$ y acidez titulable en la savia de fique (Furcraea castilla y F. macrophylla). Orinoquia 13: 5-13.

Castellanos, L., Fernández, A., Ortega, I., Soto, R. \& Martin, C. 2011. Efectividad del extracto de Furcraea hexapetala (Jacq.) Urban sobre Polyphagotarsonemus latus Banks en condiciones de laboratorio. Revista de Protección Vegetal 26: 122-124.

Cronquist, A. 1981. An integrated system of classification of flowering plants. New York Botanical Garden, New York.

Dickison, W.C. 2000. Integrative plant anatomy. Academic Press, USA.

Drummond, J.R. 1907. The literature of Furcraea with a synopsis of the known Species. Missouri Botanical Garden Annual Report, Missouri, pp. 25-75.

Evert, R.F. 2006. Esau's plant anatomy: meristems, cells, and tissues of the plant body: their structure, function, and development. John Wiley \& Sons.

Fernández, A., Castellanos, L. \& Ortega, I. 2015. Efectividad del extracto de Furcraea hexapetala (Jacq.) Urban sobre áfidos y ácaros fitófagos. Revista Científica Agroecosistemas 3: 2015.

Gañan, P. \& Mondragón, I. 2002. Surface modification of fique fibers. Effects on their physico-mechanical properties. Polymer Composites 23: 383-394.

García-Mendoza, A. 2000. Revisión taxonómica de las especies arborescentes de Furcraea (Agavaceae) en México y Guatemala. Boletín de la Sociedad Botánica de México 66: 113-129.

Guevara, S. \& Vallejo, E. 2014. Potencialidades medicinales de los géneros Furcraea y Agave. Revista Cubana de Plantas Medicinales 19: 248-263.

Heyduk, K., Mckain, M.R., Lalani, F. \& LeebensMack, J. 2016. Evolution of a CAM anatomy predates the origins of Crassulacean acid metabolism in the Agavoideae (Asparagaceae). Molecular Phylogenetics and Evolution 105: 102-113.

Iannacone, J., Cajachagua, C., Dueñas, B., Castillo, L., Alvariño, L. \& Argota, G. 2013. Toxicity of Agave americana and Furcraea andina (Asparagaceae) on Culex quinquefasciatus (Diptera) and Heleobia cumingii (Mollusca). Neotropical Helminthology 7: 311-325.

Leaf Architecture Working Group. 1999. Manual of leaf architecture: morphological description and categorization of dicotyledonous and net-veined monocotyledonous angiosperms. Smithsonian Institute, Washington, DC.

Lozano-Rivas, W.A. 2011. Uso del extracto de fique como coadyuvante de coagulación en tratamiento de efluentes de pastelería. Producción + Limpia 6: 21-34.

Lozano-Rivas, W.A. 2012. Uso del extracto de fique (Furcraea sp.) como coadyuvante de coagulación en tratamientos de lixiviados. Revista internacional de contaminación ambiental 28: 219-227.

Martínez, M.A. \& Pacheco, J.C. 2006. Protocolo para la micropropagación de Furcraea macrophylla Baker. Agronomía Colombiana 24: 207-213.
Ochoa, J.C. \& Jaramillo, L. 2007. Uso del jugo de fique como aditivo orgánico en el hormigón. Scientia et Technica 13: 455-459.

Osorio-Salazar, L., Valverde, F.A., Bonilla, C.R., Sánchez, M.S. \& Mier, C.E. 2009. Evaluación de extractos de fique, coquito, sorgo y ruda como posibles bio-herbicidas. Acta Agronómica 58: 103-108.

Peinado, J.E., Ospina, L.F., Rodríguez, L., Miller, J., Carvajal, C., Negrete, R., Cruz, D., Medina, R., Toscano, D., Toro, I., Álvarez, C.A., Serna, F., Mier, C., Mazabuel, C., Guevara, F., Porras, J.D., Gómez, J., Franco, X. \& Bonilla, C. 2006. Guía ambiental del subsector fiquero. Panamericana Formas e Impresos S.A, Bogotá, DC.

Ríos, A., García, R., García, V. \& García, C. 2017. Biorremoción de plomo en solución por fibras de Furcraea andina y biopelículas de Pseudomonas aeruginosa. Perfiles 17: 81-89.

Rivera, D., Plata, L., Castro, L., Guzmán, C. \& Escalante, H. 2012. Aprovechamiento del subproducto sólido de la digestión anaerobia del bagazo de fique (Furcraea macrophylla) para el acondicionamiento de suelos. Revista ION 25: 25-34.

Roth, I. 1976. Anatomía de las plantas superiores. Ediciones de la Biblioteca, Universidad Central de Venezuela, Caracas.

Ruzin, S.E. 1999. Plant microtechnique and microscopy. Oxford University Press, New York.

Sobrino, J., Fernández, A., Ortega, I. \& Castellanos, L. 2016. Efecto insecticida del extracto de Furcraea hexapetala (Jacq.) Urban sobre Plutella xylostella L. Centro Agrícola 43: 85-90.

Solarte, R.D. \& Osorio, O. 2014. Evaluación de la concentración del jugo de fique (Furcraea spp.) para el control In Vitro de Phytophthora infestans en plantas de papa (Solanum tuberosum L). Información Tecnológica 25: 47-54.

Solarte, R.D., Osorio, O., Hurtado, A.M. \& Mejía, D.F. 2012. Evaluación del Bioinsumo de Fique Pulverizado (Furcraea spp.) para el control in vitro de Phytophthora infestans en papa (Solanum tuberosum L). Información Tecnológica 23: 77-86.

Takhtajan, A. 2009. Flowering plants. Springer Science.

Tolivia, D. \& Tolivia, J. 1987. Fasga: A new polychromatic method for simultaneous and differential staining of plant tissues. Journal of Microscopy 148: 113-117.

La Torre, L.I., Pantoja, A.L., Mejía-España, D.F., Osorio, O. \& Hurtado, A.M. 2013. Evaluación de tratamientos térmicos para inactivación de enzimas en jugo de fique (Furcraea gigantea Vent.). Biotecnología en el Sector Agropecuario y Agroindustrial 11: 113-122.

Yeung, E.C. \& Saxena, P.K. 2005. Histological techniques. En: S. Jain, P.K. Gupta (eds.). Protocol for Somatic Embryogenesis in Woody Plants. Dordrecht: Springer, pp. 517-538.

Yokosuka, A., Sano, T., Hashimoto, K., Sakagami, H. \& Mimaki, Y. 2009. Steroidal glycosides from Furcraea foetida and their cytotoxic activity. Chemical and Pharmaceutical Bulletin 57: 1161-1166. 